\title{
Compensating for evanescent modes and estimating characteristic impedance in waveguide acoustic impedance measurements
}

Nørgaard, Kren Rahbek; Fernandez Grande, Efren

Published in:

Journal of the Acoustical Society of America

Link to article, DOI:

$10.1121 / 1.5016808$

Publication date:

2017

Document Version

Publisher's PDF, also known as Version of record

Link back to DTU Orbit

Citation (APA):

Nørgaard, K. R., \& Fernandez Grande, E. (2017). Compensating for evanescent modes and estimating characteristic impedance in waveguide acoustic impedance measurements. Journal of the Acoustical Society of America, 142(6), 3497-3509. https://doi.org/10.1121/1.5016808

\section{General rights}

Copyright and moral rights for the publications made accessible in the public portal are retained by the authors and/or other copyright owners and it is a condition of accessing publications that users recognise and abide by the legal requirements associated with these rights.

- Users may download and print one copy of any publication from the public portal for the purpose of private study or research.

- You may not further distribute the material or use it for any profit-making activity or commercial gain

- You may freely distribute the URL identifying the publication in the public portal 


\title{
Compensating for evanescent modes and estimating characteristic impedance in waveguide acoustic impedance measurements
}

\author{
Kren Rahbek Nørgaard ${ }^{a)}$ and Efren Fernandez-Grande \\ Acoustic Technology, Department of Electrical Engineering, Technical University of Denmark, \\ Ørsteds Plads, Building 352, Kongens Lyngby, DK-2800, Denmark \\ Søren Laugesen \\ Interacoustics Research Unit, Technical University of Denmark, Ørsteds Plads, Building 352, \\ Kongens Lyngby, DK-2800, Denmark
}

(Received 30 June 2017; revised 16 November 2017; accepted 20 November 2017; published online 12 December 2017)

\begin{abstract}
The ear-canal acoustic impedance and reflectance are useful for assessing conductive hearing disorders and calibrating stimulus levels in situ. However, such probe-based measurements are affected by errors due to the presence of evanescent modes and incorrect estimates or assumptions regarding characteristic impedance. This paper proposes a method to compensate for evanescent modes in measurements of acoustic impedance, reflectance, and sound pressure in waveguides, as well as estimating the characteristic impedance immediately in front of the probe. This is achieved by adjusting the characteristic impedance and subtracting an acoustic inertance from the measured impedance such that the non-causality in the reflectance is minimized in the frequency domain using the Hilbert transform. The method is thus capable of estimating plane-wave quantities of the sought-for parameters by supplying only an arbitrary initial value for the characteristic impedance. From a comparison with a simulated waveguide, it is shown that this method can accurately estimate these quantities in a waveguide that is uniform at the position of the probe. Finally, it is demonstrated how evanescent modes, characteristic impedance, and the proposed methodology can affect the measured acoustic impedance and reflectance of an occluded-ear simulator.
\end{abstract}

(C) 2017 Acoustical Society of America. https://doi.org/10.1121/1.5016808

[CAS]

Pages: 3497-3509

\section{INTRODUCTION}

In hearing diagnostics, the ear-canal acoustic impedance and reflectance can provide clues on the conductive status of the middle ear (Piskorski et al., 1999; Keefe et al., 2000; Feeney and Keefe, 2001; Keefe et al., 2012; Ellison et al., 2012; Merchant et al., 2014), and be used to calibrate stimulus levels in situ (Scheperle et al., 2008; McCreery et al., 2009; Lewis et al., 2009; Scheperle et al., 2011; Withnell et al., 2009; Souza et al., 2014). In other fields, such as musical and duct acoustics, the acoustic input impedance of wind instruments, mufflers, impedance tubes, etc., is often studied (e.g., Fletcher and Rossing, 1991; Munjal, 2014; Fletcher et al., 2005; Dalmont et al., 2012). The calculation of reflectance from a measured impedance requires that the characteristic impedance of the waveguide is known. The characteristic impedance represents the ratio of sound pressure to volume flow for a propagating plane wave in the waveguide and is inversely proportional to the crosssectional area. In ear canals, this area is difficult to measure, e.g., using ear molds or computed tomography (Egolf et al., 1993), and, in addition, vary with probe-insertion depth. A frequently used approach in hearing diagnostics is to utilize

\footnotetext{
a) Also at: Interacoustics A/S, Audiometer Allé 1, Middelfart, DK-5500, Denmark. Electronic mail: krng@interacoustics.com
}

an invariant value for the characteristic impedance, corresponding to an average adult ear canal, and another value for infants. However, this uncertainty in characteristic impedance inevitably introduces errors into the ear-canal reflectance.

The acoustic input impedance of a waveguide is defined as the ratio of acoustic pressure to volume flow in a crosssectional plane. The sought-for parameter is thus the planewave impedance, but it is difficult to accurately control such uniform acoustic flow and measure the sound pressure simultaneously. Acoustic impedance is often measured by means of an acoustic probe that, based on a preceding calibration procedure, injects a known acoustic volume flow into a waveguide through a speaker-tube aperture in this plane. At the same time, the sound pressure is measured across another aperture in that plane using a microphone. The geometrical mismatch between the speaker tube and waveguide causes higher-order modes to be excited in the waveguide. These higher-order modes describe variations in the sound pressure across the radial and azimuthal axes of the waveguide, as opposed to plane waves which only vary along the axis of propagation in the lossless case. If the excitation frequency is below the cut-on frequency for any given higher-order mode (i.e., the frequency at which the mode starts to propagate), that mode will be exponentially decaying (evanescent) along the axis of the waveguide. With the speaker and 
microphone tubes positioned in the same plane, the resulting impedance measurement will be affected by some disturbance, in addition to the plane-wave sound field, described by these evanescent modes, as there is no spatial decay. A measured acoustic impedance comprises the superposition of impedance characteristics of each excited mode. For frequencies high enough that higher-order modes start to propagate and are reflected by a waveguide termination, the interpretability of the measured impedance is limited.

\section{A. Existing methods for estimating characteristic impedance}

Keefe et al. (1992), Huang et al. (2000), and Keefe and Abdala (2007) showed that the characteristic impedance of a waveguide can be calculated by averaging the real part of the input impedance across frequency. Keefe et al. (1992) evaluated the capability of this method in estimating the characteristic impedance of different waveguides and observed errors in the range of 3\%-6\%. However, their evaluation does not take into account the effect of finite frequency bandwidth in estimating the characteristic impedance of shorter waveguides. With their chosen waveguide lengths $(34.8-51.8 \mathrm{~cm})$, the input impedances contain a large part of the resonant behavior, with multiple maxima throughout the investigated frequency range $(0.1-10.7 \mathrm{kHz})$, and the real impedance average provides a relatively good estimate of characteristic impedance. With the typical length of human ear canals ( $l \approx 2.5 \mathrm{~cm}$, minus probe insertion), the ear-canal impedance has none or few impedance maxima within their investigated frequency range. The method is thus very sensitive to these impedance maxima being included in or excluded from the average. In addition, the method cannot account for any frequency dependency in the termination impedance of a waveguide, which will inherently have an effect on averaging the real part of the impedance across a limited bandwidth.

Rasetshwane and Neely (2011) and Rasetshwane et al. (2012) calculated the time-domain reflectance, the inverse Fourier transform of reflectance, and used it to estimate the characteristic impedance by iteratively adjusting it to minimize the reflection at time $t=0$. They did, however, neither take evanescent modes nor the smearing of the time-domain reflectance as a result of frequency-domain windowing into account. They also made the observation that time-domain reflectance was non-causal, referring to this as a measurement artifact, and carried out a "time-reversed addition," thus removing the artifact. It is possible that this artifact could originate from a combination of evanescent modes affecting the measurement and calibration errors in the Thévenin source parameters due to the presence of evanescent modes during calibration (Siegel and Neely, 2017; Nørgaard et al., 2017). These errors likely introduce an additional error into the estimated characteristic impedance.

\section{B. Existing methods for accounting for evanescent modes}

Keefe et al. (1992), Huang et al. (2000), Voss and Allen (1994), and Siegel and Neely (2017) protruded the microphone tube beyond the speaker tube in order to eliminate the contribution from evanescent modes to the measured probe pressures during calibration and measurements. However, this approach includes the excess piece of waveguide between the speaker and microphone tubes in the source parameters. Thus, errors are introduced into subsequent measurements in waveguides of radii different from the calibration waveguides (Huang et al., 2000), since the characteristic impedance of this piece of waveguide depends on the waveguides radius.

Keefe and Benade (1981) and Fletcher et al. (2005) showed that evanescent modes can be approximated by the addition of an acoustic inertance to the plane-wave acoustic input impedance and calculated analytically for a known probe geometry and waveguide radius. The approximation as an inertance is valid up to some point in frequency, below the cut-on frequency of the lowest-order non-uniform mode, and shifts the position of impedance minima in frequency while maintaining the position of maxima. However, other effects can also contribute to such series inertance, e.g., a horn gradient (Rasetshwane and Neely, 2011).

Brass and Locke (1997) investigated the effect of evanescent modes on ear-canal sound-pressure measurements. They predicted the contribution from the evanescent-modes inertance for various probe and waveguide configurations and showed that this introduces errors up to $3 \mathrm{~dB}$ below $10 \mathrm{kHz}$ into measurements of sound pressure in adult ear canals. On the other hand, Schmidt and Hudde (2009) showed that evanescent modes can introduce large errors into ear-canal impedance and reflectance measurements above $3 \mathrm{kHz}$. They also attempted to compensate for the effect using an acoustic inertance and the method of Karal (1953), although this requires information about area discontinuity and thus the ear-canal geometry.

Fletcher et al. (2005) used an acoustic probe, delivering an assumed constant acoustic volume flow through a narrow annulus, to measure acoustic impedance. From an impedance measurement in a circular uniform waveguide of known radius, they added an acoustic inertance in series with the measured impedance and adjusted its magnitude such that impedance minima were translated to the halfway point between maxima. With this procedure, the probe and the obtained evanescent-modes inertance could be utilized in subsequent measurements for estimating the plane-wave impedance in a waveguide of identical radius at the position of the probe. Their methodology is thus not applicable in ear-canal measurements, as the ear canal is not necessarily circular and the cross-sectional area is typically not known.

\section{Proposed compensation methodology}

There is thus a need for a fast, non-invasive method that can accurately estimate the characteristic impedance at the position of the acoustic probe and the inertance that accounts for evanescent modes in a waveguide of unknown area. This paper proposes a method capable of estimating the size of this inertance and the characteristic impedance from a measurement of acoustic input impedance using an acoustic probe. The effects of evanescent modes and unknown 
characteristic impedance on the measured impedance and reflectance are thus compensated for. The method is at least applicable in waveguides that are uniform at the position of the probe, i.e., unaffected by a horn inertance (Rasetshwane and Neely, 2011), but the waveguide can otherwise possess an arbitrary area function and entryway geometry. The proposed method utilizes the relationship between the real and imaginary parts of a causal function using the Hilbert transform. The evanescent-modes inertance and characteristic impedance are adjusted to minimize the non-causality in the measured reflectance. In this way, the proposed method differs from existing methods in that it takes both the effects of evanescent modes and characteristic impedance into account. Thus, the estimated characteristic impedance is not affected by errors due to evanescent modes and vice versa, although the method does rely on the ability of an inertance in characterizing the non-causality in a given impedance measurement. In addition, errors due to finite measurement spectra are avoided by truncating the Hermitian-symmetric, harmonic reflectance spectrum to restore differentiability.

\section{METHODS}

\section{A. Quantifying non-causality using the Hilbert transform}

A causal system is independent of future inputs and quantities describing the behavior of passive, linear, physical, input-output systems must conform to this property. Assessing the causality of a quantity, characterizing a given physical system, can provide information on the physical validity of such quantity or phenomena that introduce noncausality into such quantity. Causality of a function can intuitively be assessed in the time domain, but it is also possible to investigate this property in the frequency domain using the Hilbert transform (Papoulis, 1962; Hsu, 1967). The Hilbert transform of a real-valued function of time $\mathcal{H}[h(t)]$ is defined as the convolution of the function with $1 /(\pi t)$,

$$
\mathcal{H}[h(t)]=\frac{1}{\pi t} * h(t),
$$

where the asterisk denotes convolution. Since the Fourier transform $\mathcal{F}[1 /(\pi t)]=-j \operatorname{sgn} \omega$, the Hilbert transform introduces a phase shift of -90 degrees into its argument. $j$ is the unit-imaginary number, $\operatorname{sgn}(\cdot)$ is the signum function, and $\omega$ is the angular frequency. For a causal function, $h(t<0)=0$, the real $\operatorname{Re}\{\cdot\}$ and imaginary $\operatorname{Im}\{\cdot\}$ parts of the Fourier transform of that function, $\mathcal{F}[h(t)]=H(\omega)$, are related by the Hilbert transform and the behavior in $\operatorname{Re}\{H(\infty)\} \equiv \lim _{\omega \rightarrow \infty} \operatorname{Re}\{H(\omega)\}$ (Papoulis, 1962),

$$
\begin{aligned}
& \operatorname{Re}\{H(\omega)\}=\operatorname{Re}\{H(\infty)\}+\mathcal{H}[\operatorname{Im}\{H(\omega)\}], \\
& \operatorname{Im}\{H(\omega)\}=\mathcal{H}^{-1}[\operatorname{Re}\{H(\omega)\}] .
\end{aligned}
$$

Here, $h(t)=\operatorname{Re}\{H(\infty)\} \delta(t)+h(t>0), \delta(\cdot)$ is the Diracdelta function, and $\operatorname{Im}\{H(\infty)\}=0$. An estimate of the Fourier transform of the function $\hat{H}(\omega)$ can be obtained from $\operatorname{Re}\{H(\infty)\}$ and the Hilbert pairs,

$$
\begin{aligned}
\hat{H}(\omega)= & \operatorname{Re}\{H(\infty)\}+\mathcal{H}[\operatorname{Im}\{H(\omega)\}] \\
& +j \mathcal{H}^{-1}[\operatorname{Re}\{H(\omega)\}] .
\end{aligned}
$$

The estimation error $\epsilon_{H}$ can then be assessed by selecting an initial value for $\operatorname{Re}\{H(\infty)\}$, which might not be known due to the practical limitation of finite frequency range,

$$
\epsilon_{H}(\omega)=H(\omega)-\hat{H}(\omega) .
$$

This error quantifies any non-causal component in $H(\omega)$ and error in $\operatorname{Re}\{H(\infty)\}$. In this study, phenomena that introduce non-causality into measured quantities will be quantified by the real and imaginary estimation errors

$$
\begin{aligned}
\operatorname{Re}\left\{\epsilon_{H}(\omega)\right\}= & \operatorname{Re}\{H(\omega)\}-\operatorname{Re}\{H(\infty)\} \\
& -\mathcal{H}[\operatorname{Im}\{H(\omega)\}], \\
\operatorname{Im}\left\{\epsilon_{H}(\omega)\right\}= & \operatorname{Im}\{H(\omega)\}-\mathcal{H}^{-1}[\operatorname{Re}\{H(\omega)\}] .
\end{aligned}
$$

\section{B. Application of the Hilbert transform to waveguide acoustic input impedance}

Acoustic impedance is inherently causal and defined as the ratio of acoustic pressure to volume flow. In its timedomain representation, an acoustic waveguide yields an immediate pressure response at $t=0$, equal to the characteristic impedance, to such unit-delta volume-flow excitation (Keefe et al., 1992; Huang et al., 2000; Keefe and Abdala, 2007). This gives rise to the property that the plane-wave impedance $Z_{\mathrm{pw}}$ of an acoustic waveguide converges to the lossless characteristic impedance in the limit $\operatorname{Re}\left\{Z_{\mathrm{pw}}(\infty)\right\}=Z_{0}$. As a result of this property, the real and imaginary estimation errors in Eqs. (6) and (7) can be written in terms the plane-wave impedance and both yield zero,

$$
\begin{aligned}
& \operatorname{Re}\left\{\epsilon_{Z_{\mathrm{pw}}}\right\}=\operatorname{Re}\left\{Z_{\mathrm{pw}}\right\}-Z_{0}-\mathcal{H}\left[\operatorname{Im}\left\{Z_{\mathrm{pw}}\right\}\right]=0, \\
& \operatorname{Im}\left\{\epsilon_{Z_{\mathrm{pw}}}\right\}=\operatorname{Im}\left\{Z_{\mathrm{pw}}\right\}-\mathcal{H}^{-1}\left[\operatorname{Re}\left\{Z_{\mathrm{pw}}\right\}\right]=0 .
\end{aligned}
$$

$Z_{0}$ might not be known; however, an arbitrary initial value $Z_{0}^{\prime}=\rho c / A$ can be chosen, representing the cross-sectional area $A$, air density $\rho$, and speed of sound $c$. In this study, circular waveguides are considered and from the radius $a$, $A=\pi a^{2}$. The real impedance estimation error $\operatorname{Re}\left\{\epsilon_{Z_{\mathrm{pw}}}\right\}$ now yields the error in the chosen initial value $Z_{0}^{\prime}$ and the characteristic impedance can be estimated,

$$
\operatorname{Re}\left\{\epsilon_{Z_{\mathrm{pw}}}\right\}+Z_{0}^{\prime}=\hat{Z}_{0} .
$$

If the input impedance of a waveguide is measured using an acoustic probe, this measured impedance $Z_{\text {meas }}$ can be regarded as the ratio of measured sound pressure at the probe microphone $P_{\text {meas }}$, to the volume flow injected by the probe speaker $U$ in the plane of the probe tip. The measurement will thus be affected by evanescent modes which can be approximated by the impedance of a series acoustic inertance $L$ to the plane-wave impedance $Z_{\mathrm{pw}}$ (Keefe and Benade, 1981; Fletcher et al., 2005),

$$
Z_{\text {meas }}=\frac{P_{\text {meas }}}{U} \simeq Z_{\mathrm{pw}}+j \omega L
$$


The approximation of evanescent modes as an inertance is only valid up to a certain point below the frequency where the first non-uniform mode starts to propagate. For a circular waveguide, these frequencies are given by (Blackstock, 2000)

$$
\omega_{m n}=\frac{\alpha_{m n}^{\prime} c}{a},
$$

where $\alpha_{m n}^{\prime}$ is the $n$th zero of the cylindrical bessel-function derivative of the first kind $J_{m}^{\prime}$ of order $m$. The present analysis is based on the assumption that the approximation as an inertance applies across the infinite frequency spectrum. For a band-limited measurement, such ideal acoustic inertance introduces a non-causality into the measured impedance, presumably due to $\operatorname{Im}\left\{Z_{\text {meas }}(\infty)\right\} \neq 0$. In practice, the addition to the plane-wave impedance, constituted by higherorder modes, will exhibit a resonant behavior above the cuton frequency and eventually satisfy $\operatorname{Im}\left\{Z_{\text {meas }}(\infty)\right\}=0$. Since the Hilbert transform is an additive operator, and given the purely imaginary addition of the approximation of evanescent modes, the imaginary impedance estimation error yields the evanescent-modes inertance when this approximation of $Z_{\text {meas }}$ is inserted into Eq. (9),

$$
\operatorname{Im}\left\{\epsilon_{Z_{\text {meas }}}\right\}=\omega L \text {. }
$$

However, the evanescent-modes inertance introduces an error into the characteristic-impedance estimation in Eq. (10) such that,

$$
\operatorname{Re}\left\{\epsilon_{Z_{\text {meas }}}\right\}+Z_{0}^{\prime}=\hat{Z}_{0}-\mathcal{H}[\omega L] .
$$

This is the remainder of the imaginary term $j \omega L$ when inserting $Z_{\text {meas }}$ into Eq. (8). The additional term $-\mathcal{H}[\omega L]$ is undefined and diverges to positive infinity for all $\omega$ in the limit of the Hilbert-transform convolution integral over an infinite spectrum. However, estimating the plane-wave impedance $\hat{Z}_{\mathrm{pw}}$ by subtracting the evanescent-modes inertance from the measured impedance,

$$
\hat{Z}_{\mathrm{pw}}=Z_{\text {meas }}-j \omega L
$$

the characteristic impedance can be estimated from Eq. (10).

\section{Application of the Hilbert transform to waveguide reflectance measurements}

In practice, acoustic impedance is measured in discrete points over a finite range of frequencies. The inherent nonsmooth behavior of the real and imaginary parts and the divergent behavior in the imaginary part as $\omega \rightarrow 0$ of the acoustic input impedance of a non-vented waveguide, such as the ear canal, can introduce instabilities into the calculation of the discrete Hilbert transform. It is therefore convenient to evaluate the discrete Hilbert transform of the real and imaginary parts of acoustic reflectance $R$ rather than impedance. The reflectance of an acoustic waveguide can be regarded as the ratio of incident to reflected pressure waves, in the continuous-time domain equivalent to the response to an incident Dirac-delta pressure impulse. Therefore, no reflection can occur at or before this impulse and the reflectance is strictly causal, i.e., no impulses at the origin
$\left(\operatorname{Re}\left\{R_{\mathrm{pw}}(\infty)\right\}=0\right)$, if the incident and reflected waves are planar and the acoustic waveguide is uniform at the position of the measurement. In general terms, any non-causality in the acoustic impedance $Z$, and thus represented by the impedance estimation error $\epsilon_{Z}$, is equivalently observable in the reflectance $R$. However the initial value $Z_{0}^{\prime}$ must be utilized to compute reflectance,

$$
R=\frac{Z-Z_{0}^{\prime}}{Z+Z_{0}^{\prime}}
$$

The reflectance estimation error can now be defined,

$$
\epsilon_{R}=R-\mathcal{H}[\operatorname{Im}\{R\}]-j \mathcal{H}^{-1}[\operatorname{Re}\{R\}] .
$$

Section II B showed that the impedance estimation error $\epsilon_{Z}$ essentially represents the impedance of the non-causal component in the measured impedance and its negative Hilbert-transform pairs. The impedance and reflectance estimation errors $\epsilon_{Z}$ and $\epsilon_{R}$ can also be defined from from the negative-time parts of the time-domain impedance $z(t)$ and reflectance $r(t)$,

$$
\begin{aligned}
\epsilon_{Z} & =\mathcal{F}[z(t) 2 \theta(-t)], \\
\epsilon_{R} & =\mathcal{F}[r(t) 2 \theta(-t)],
\end{aligned}
$$

where $\theta(\cdot)$ is the Heaviside step function. While a noncausal series component is simply added to the impedance in the time domain, additional delayed components are introduced into the reflectance. This is a result of the bilinear transform, used for converting between impedance and reflectance [Eq. (16)], not being an additive operation. However, it is only the non-causal component in the impedance that can cause a response to the incident pressure pulse and contribute to the negative-time part of the time-domain reflectance. The reflectance estimation error $\epsilon_{R}$ then similarly represents the reflectance of such non-causal component and its negative Hilbert-transform pairs, for the arbitrary characteristic-impedance initial value $Z_{0}^{\prime}$. For an evanescentmodes inertance, this results in the reflectance of $j \omega L-\mathcal{H}[\omega L]$, assuming that this Hilbert transform is defined. The impedance estimation error $\epsilon_{Z}$ can then be calculated from the reflectance estimation error $\epsilon_{R}$ using the bilinear transform. However, if $Z_{0}^{\prime} \neq Z_{0}$,

$$
\operatorname{Re}\{R(\infty)\}=\frac{Z_{0}-Z_{0}^{\prime}}{Z_{0}+Z_{0}^{\prime}} \neq 0 .
$$

This is accounted for by adding the behavior of $Z_{0}^{\prime}$ to $\epsilon_{Z}$ in its relation with $\epsilon_{R}$,

$$
\begin{aligned}
\epsilon_{R} & =\frac{\left[\epsilon_{Z}+Z_{0}^{\prime}\right]-Z_{0}^{\prime}}{\left[\epsilon_{Z}+Z_{0}^{\prime}\right]+Z_{0}^{\prime}}=\frac{\epsilon_{Z}}{\epsilon_{Z}+2 Z_{0}^{\prime}}, \\
\epsilon_{Z} & =\frac{2 Z_{0}^{\prime} \epsilon_{R}}{1-\epsilon_{R}} .
\end{aligned}
$$

The procedures in Sec. II B can now be employed to investigate the impedance estimation error $\epsilon_{Z}$. From the estimated characteristic impedance $\hat{Z}_{0}$ and plane-wave impedance $\hat{Z}_{\mathrm{pw}}$, the plane-wave reflectance $\hat{R}_{\text {pw }}$ can be estimated, 


$$
\hat{R}_{\mathrm{pw}}=\frac{\hat{Z}_{\mathrm{pw}}-\hat{Z}_{0}}{\hat{Z}_{\mathrm{pw}}+\hat{Z}_{0}} .
$$

Having estimated the plane-wave impedance, it can be used to estimate the plane-wave sound pressure $\hat{P}_{\mathrm{pw}}$ as well. This is possible since the ratio of compensated to noncompensated impedances $\hat{Z}_{\mathrm{pw}}$ and $Z_{\text {meas }}$ yields the ratio of sound pressure on the probe microphone affected by evanescent modes $P_{\text {meas }}$ and the plane-wave sound pressure at the position of the probe

$$
\hat{P}_{\mathrm{pw}}=P_{\text {meas }} \frac{\hat{Z}_{\mathrm{pw}}}{Z_{\text {meas }}} .
$$

This estimated plane-wave sound pressure can then be utilized, in combination with the estimated plane-wave impedance and characteristic impedance, or reflectance, for calibrating stimulus levels.

\section{Harmonic Hilbert spectra and truncation}

The Hilbert transform of the real or imaginary part of a Hermitian-symmetric, harmonic spectrum is usually calculated as a circular convolution of the argument with $1 /(\pi \omega)$ using the forward and inverse Fourier transforms,

$$
\mathcal{H}[\operatorname{Re}\{H(\omega)\}]=\mathcal{F}^{-1}[\mathcal{F}[\operatorname{Re}\{H(\omega)\}] \mathcal{F}[1 /(\pi \omega)]] .
$$

When $1 /(\pi \omega)$ is replicated at the integer multiples of the sampling frequency $\omega_{\mathrm{s}}$ and superimposed, it converges to a cotangent,

$$
\sum_{n=-\infty}^{\infty} \frac{1}{\pi\left(\omega-n \omega_{\mathrm{s}}\right)}=\frac{\cot \left[\pi \omega / \omega_{\mathrm{s}}\right]}{\omega_{\mathrm{s}}} .
$$

The Hilbert-transform convolution integral can then be thought of as bounded by the positive and negative Nyquist frequencies, i.e., half the sampling frequency $\omega_{\mathrm{s}} / 2$,

$$
\mathcal{H}[\operatorname{Re}\{H(\omega)\}]=\int_{-\omega_{\mathrm{s}} / 2}^{\omega_{\mathrm{s}} / 2} \frac{\cot \left[\pi(\omega-\tau) / \omega_{\mathrm{s}}\right]}{\omega_{\mathrm{s}}} \operatorname{Re}\{H(\tau)\} \mathrm{d} \tau .
$$

In order for the circular Hilbert transform to yield accurate results, the harmonic spectrum of its argument must be differentiable at the Nyquist frequency. This is the frequency from which the negative-frequency part of the spectrum is replicated to obtain a harmonic spectrum when calculating the Hilbert transform of the real and imaginary parts and the inverse Fourier transform of the spectrum. A nondifferentiable spectrum causes a divergent behavior in the Hilbert transform towards the Nyquist frequency. A spectrum is differentiable if the Nyquist frequency coincides with an ordinate zero crossing of the imaginary part and a local extreme of the real part of the spectrum, but this is an unlikely case for a measured or modeled reflectance across a fixed frequency range. In addition, due to anti-aliasing filters in the data-acquisition system and potential limitations in the bandwidth of the calibration data, reflectance measurements will only be valid up to a certain maximum frequency below the
Nyquist frequency $\omega_{\mathrm{m}}<\omega_{\mathrm{s}} / 2$. The data above $\omega_{\mathrm{m}}$ can thus not be utilized for the analysis. However, differentiability can be restored by adaptively truncating the spectrum such that this truncation frequency $\omega_{\mathrm{t}} \leq \omega_{\mathrm{m}}$ coincides with such points that restore differentiability in the real and imaginary parts of the harmonic reflectance spectrum. This process discards some high-frequency data from the spectrum for the calculation of the Hilbert transform and inverse Fourier transform, but yields a significant increase in the validity of the real and imaginary estimation errors. It also facilitates the assessment of time-domain reflectance without windowing as the discretized sinc-function impulse response of the ideal low-pass filter, that the harmonic spectrum is implicitly multiplied by in the inverse Fourier transform, coincides with the zeros $\operatorname{sinc}(\pi n)=0$ for $n \neq 0$ and $\operatorname{sinc}(\pi n)=1$ for $n=0$, where $n$ is the integer sample number.

For a harmonic spectrum, the impedance of the evanescent-modes inertance $Z_{L}$ can be regarded as an imaginary sawtooth function with zero mean, passing though the origin, with ridges at the odd truncation-frequency integer multiples, and slope $L$,

$$
Z_{L}=j L\left(\frac{\omega}{2 \omega_{\mathrm{t}}}-\left\lfloor\frac{\omega}{2 \omega_{\mathrm{t}}}-\frac{1}{2}\right\rfloor-1\right),
$$

where $\lfloor\cdot\rfloor$ denotes flooring to nearest integer. The Hermitiansymmetric, harmonic spectrum of the impedance of an acoustic inertance will thus contain a non-differentiable component at the truncation frequency due to the complexconjugate mirrored part of the spectrum. Figure 1(a) shows the reflectance of a four-sample delay line $R_{\mathrm{pw}}$ corresponding to a uniform, lossless, rigidly terminated waveguide of length $l=c / \omega_{\mathrm{t}}$. The figure also shows the effect of an evanescent-modes inertance on reflectance $R_{\text {meas }}$ with $L /\left(\omega_{\mathrm{t}} Z_{0}\right)=1$, and added to the impedance [Eq. (11)] of the delay line before calculating reflectance. The impulse delay of an even number of samples results in a differentiable spectrum at $\omega_{\mathrm{t}}$ regardless of evanescent modes, whereby $\omega_{\mathrm{t}}=\omega_{\mathrm{m}}=\omega_{\mathrm{s}} / 2$. The figure shows how the evanescentmodes inertance translates in frequency both the real and imaginary parts of reflectance, corresponding to impedance minima. Notice how the differentiable reflectance spectrum effectively causes a smooth transition from the original to the replicated spectrum at the truncation frequency. Figure 1(b) shows the impedance of the harmonic evanescentmodes inertance $Z_{L}$, its negative Hilbert transform $-\mathcal{H}\left[\operatorname{Im}\left\{Z_{L}\right\}\right]$, and the real and imaginary impedance estimation errors $\operatorname{Re}\left\{\epsilon_{Z}\right\}$ and $\operatorname{Im}\left\{\epsilon_{Z}\right\}$, calculated from reflectance [Eq. (22)]. The figure illustrates how this smooth transition in the reflectance spectrum results in a smoothing of the sawtooth function and thus an error in the estimation of the evanescent-modes inertance towards $\omega_{\mathrm{t}}$. An approach is therefore employed which estimates the evanescent-modes inertance $\hat{L}$ by adjusting it such that the absolute imaginary impedance estimation error, averaged across frequency, $\left|\left\langle\operatorname{Im}\left\{\epsilon_{Z}\right\}\right\rangle\right|$ is minimized. Subsequently, the characteristic impedance $\hat{Z}_{0}$ is estimated by averaging the sum of the real impedance estimation error and the characteristic-impedance 
(a)

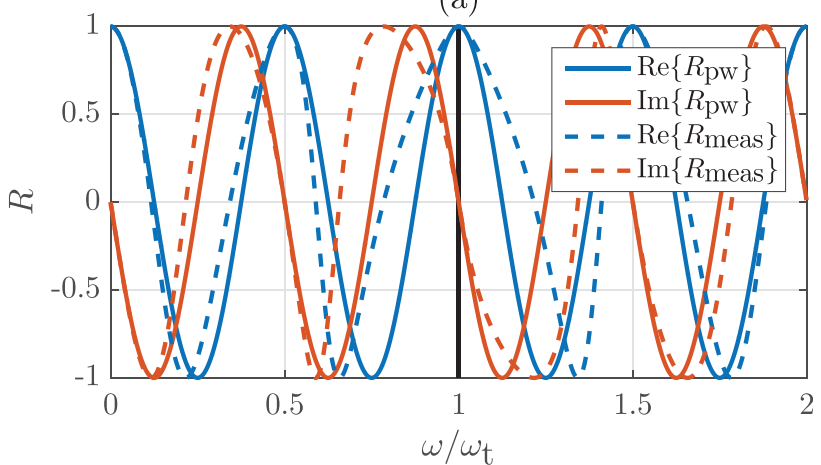

(b)

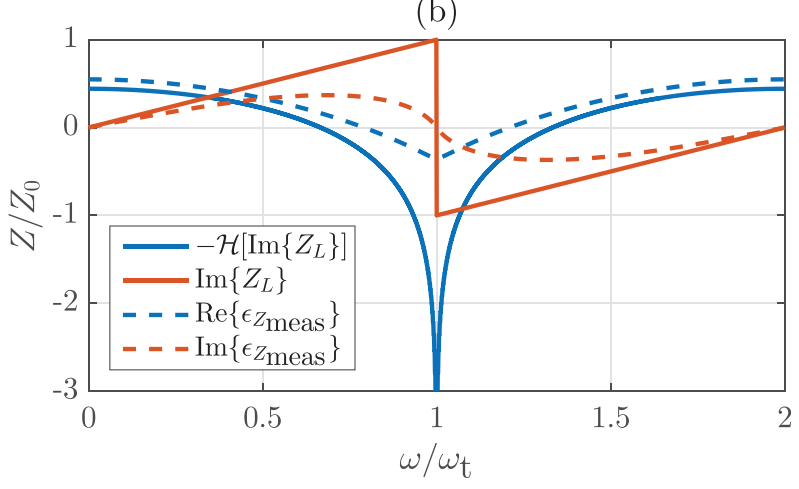

FIG. 1. (Color online) (a) The real and imaginary parts of the reflectance of an ideal four-sample delay line $R_{\mathrm{pw}}$, and including the simulated effect of evanescent modes $R_{\text {meas }}$ using an inertance $L /\left(\omega_{\mathrm{t}} Z_{0}\right)=1$. (b) The imaginary part of the impedance of the evanescent-modes inertance $\operatorname{Im}\left\{Z_{L}\right\}$ and its negative Hilbert transform $-\mathcal{H}\left[\operatorname{Im}\left\{Z_{L}\right\}\right]$. The real and imaginary impedance estimation errors $\operatorname{Re}\left\{\epsilon_{Z_{\text {meas }}}\right\}$ and $\operatorname{Im}\left\{\epsilon_{Z_{\text {meas }}}\right\}$, derived from reflectance [Eq. (22)]. The quantities are normalized with respect to an arbitrary characteristic impedance $Z_{0}$ and truncation frequency $\omega_{\mathrm{t}}$.

initial value across frequency $\left\langle\operatorname{Re}\left\{\epsilon_{Z}\right\}+Z_{0}^{\prime}\right\rangle$. Averaging the quantities minimizes the influence of potential noise and other measurement artifacts on the estimated quantities. The error introduced into the characteristic-impedance estimate due to evanescent modes $-\mathcal{H}\left[\operatorname{Im}\left\{Z_{L}\right\}\right]$ does not diverge to infinity in case of a harmonic frequency spectrum and smoothed evanescent-modes sawtooth function, as Fig. 1(b) also illustrates. At $\omega=0$ in Eq. (27), where discontinuities in the cotangent intersect abscissa crossings in the smoothed sawtooth function, the convolution results in an offset. At the truncation frequency $\omega=\omega_{t}$, where discontinuities in the cotangent intersect the smoothed ridges in the sawtooth function, the convolution diverges to a finite value of opposite sign of operation.

\section{E. Summary of the proposed compensation scheme}

The compensation scheme of the proposed methodology for estimating the evanescent-modes inertance $\hat{L}$ and characteristic impedance $\hat{Z}_{0}$ can be summarized to consist of the following three steps:

(1) Measuring the impedance affected by evanescent modes $Z_{\text {meas }}$ of a waveguide in the usual way using a Thévenincalibrated acoustic probe. Calculating the reflectance affected by evanescent modes $R_{\text {meas }}^{\prime}$, where the prime superscript indicates that it was calculated from the characteristic-impedance initial value $Z_{0}^{\prime}$.
(2) Estimating the evanescent-modes inertance $\hat{L}$ by adjusting $L$ in Eq. (15) such that the absolute averaged imaginary impedance estimation error $\operatorname{Im}\left\{\epsilon_{Z}\right\}$, calculated from Eq. (22), is minimized,

$$
\hat{L}=\underset{L}{\arg \min }\left|\left\langle\operatorname{Im}\left\{\epsilon_{Z}\right\}\right\rangle\right| \text {. }
$$

The plane-wave impedance $\hat{Z}_{\mathrm{pw}}$ can then be estimated from Eq. (15), and the plane-wave reflectance $\hat{R}_{\mathrm{pw}}^{\prime}$, still using the characteristic-impedance initial value $Z_{0}^{\prime}$, from Eq. (23).

(3) Estimating the characteristic impedance $\hat{Z}_{0}$ as the averaged sum of the real impedance estimation error $\operatorname{Re}\left\{\epsilon_{Z}\right\}$ and characteristic-impedance initial value $Z_{0}^{\prime}$,

$$
\hat{Z}_{0}=\left\langle\operatorname{Re}\left\{\epsilon_{Z}\right\}+Z_{0}^{\prime}\right\rangle \text {. }
$$

The plane-wave reflectance $\hat{R}_{\text {pw }}$ can then be estimated from Eq. (23).

The truncation procedure was performed in each iteration of adjusting $L$ in Eq. (29) of the compensation scheme, as Fig. 1(a) indicated that a change in $L$ can affect the highestpossible frequency at which differentiability is restored. Due to the frequency spectrum being discrete, it is not possible to achieve perfect differentiability. The parts of the Hilberttransform spectra close to the truncation frequency are therefore omitted from the averages $\left\langle\operatorname{Im}\left\{\epsilon_{Z}\right\}\right\rangle$ and $\left\langle\operatorname{Re}\left\{\epsilon_{Z}\right\}+Z_{0}^{\prime}\right\rangle$.

\section{F. Equipment and measurements}

The measurements reported in this study were carried out using a FireFace UC sound card (RME Audio, Haimhausen, Germany) controlled through custom-written MATLAB (The MathWorks, Inc., Natick, MA) software and the third-party utility Playrec. ${ }^{1}$ The Hilbert function, as part of the MATLAB Signal Processing Toolbox, was used to calculate discrete Hilbert transforms and time-domain reflectances were calculated using only the ifft function, both from the Hermitian-symmetric, truncated spectra. A Titan-based ear probe (Interacoustics A/S, Middelfart, Denmark) was used, but modified to improve the high-frequency performance via internal crosstalk reduction.

Probe pressures were obtained by applying a frequencyequalized wideband chirp to the probe so as to provide a flat probe pressure in a non-reflecting load of radius similar to an adult ear canal. The chirp was played back in the probe in phase-locked blocks with a length of $N=2048$ samples at a sampling rate of $f_{\mathrm{s}}=44.1 \mathrm{kHz}$ which were each recorded using the probe microphone and averaged to reduce the noise in the measurements. Prior to the measurement, the probe was calibrated to obtain its Thévenin-equivalent source parameters, the source pressure $P_{\mathrm{s}}$ and source impedance $Z_{\mathrm{s}}$, and facilitate the measurement of acoustic impedance using the calibration method described by Nørgaard et al. (2017). The measured impedance $Z_{\text {meas }}$ was calculated from the measured probe pressure $P_{\text {meas }}$ and the Thévenin source parameters,

$$
Z_{\text {meas }}=Z_{\mathrm{s}} \frac{P_{\text {meas }}}{P_{\mathrm{s}}-P_{\text {meas }}} .
$$


The calibration waveguides were of lengths $l=1.2,1.45$, 1.75 , and $2 \mathrm{~cm}$ and radius $a=2 \mathrm{~mm}$. For applying the proposed methodology, it is important that the calibration method characterizes the probe in a way such that the measured impedance can be interpreted as in Eq. (11), and not including any parallel components such as a compliance, as demonstrated by Nørgaard et al. (2017). The reflectance cannot be measured at zero frequency, but this value is required for calculating the Hilbert and Fourier transforms, and it was therefore manually set to $R(f=0)=1$. The maximum calibration frequency, and thereby maximum valid measurement point, was $f_{\mathrm{m}}=20 \mathrm{kHz}$.

An evaluation waveguide of radius $a=2.35 \mathrm{~mm}$ and length $l=2.8 \mathrm{~cm}$ was used to evaluate the proposed method. The evaluation waveguide was constructed similar to the calibration waveguides used by Nørgaard et al. (2017), inserting the probe using the same hard-rubber adapter and thus positioning the probe tube flush in the input plane. The radius was chosen to deviate from the calibration waveguides and still expose a small part of the rubber section to the acoustic domain. The length was chosen to better separate features apart in the time domain.

In addition, a type 4157 occluded-ear simulator (Brüel \& Kjær Sound \& Vibration A/S, Nærum, Denmark), typically designated a 711 coupler, was used to further evaluate the proposed methodology in a load similar to the human ear canal. A 3D-printed part was attached to the ear simulator that uniformly extended its length, as the non-removable protection grid just in front of the reference plane in the ear simulator caused errors into the estimation of characteristic impedance. The ear simulator had the following dimensions: radius $a=3.75 \mathrm{~mm}$ and length $l=2.25 \mathrm{~cm}$, terminated by a reference microphone. The probe was inserted into the ear simulator using a standard, mushroom-shaped, yellow, $8 \mathrm{~mm}$ rubber ear tip (Sanibel Supply, Middelfart, Denmark). The evanescent-modes inertances obtained in the evaluation waveguide and ear simulator were compared to analytically calculated values for the specific probe-waveguide configuration, using the approach of Nørgaard et al. (2017).

\section{SIMULATIONS}

To analytically investigate the effects of characteristic impedance and evanescent modes on the causality of reflectance, given the approximation of an acoustic inertance [Eq. (11)], the analytical plane-wave input impedance of a rigidly terminated, uniform waveguide was calculated using a transmission-line model,

$$
Z_{\mathrm{pw}}=Z_{0, \mathrm{loss}} \operatorname{coth} \Gamma l .
$$

$\Gamma$ is the propagation constant and $Z_{0, \text { loss }}$ is the frequencydependent characteristic impedance, both including thermoviscous losses and calculated from Keefe (1984). Calculating the reflectance, it can be simplified,

$$
R_{\mathrm{pw}}=e^{-2 \Gamma l} \text {. }
$$

$\Gamma$ is mainly imaginary and the real and imaginary parts of the plane-wave reflectance $R_{\mathrm{pw}}$ are thus given by a cosine

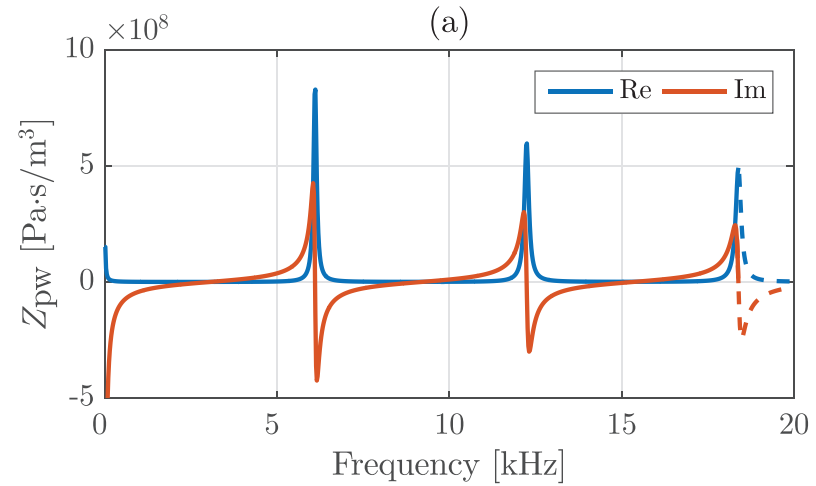

(b)

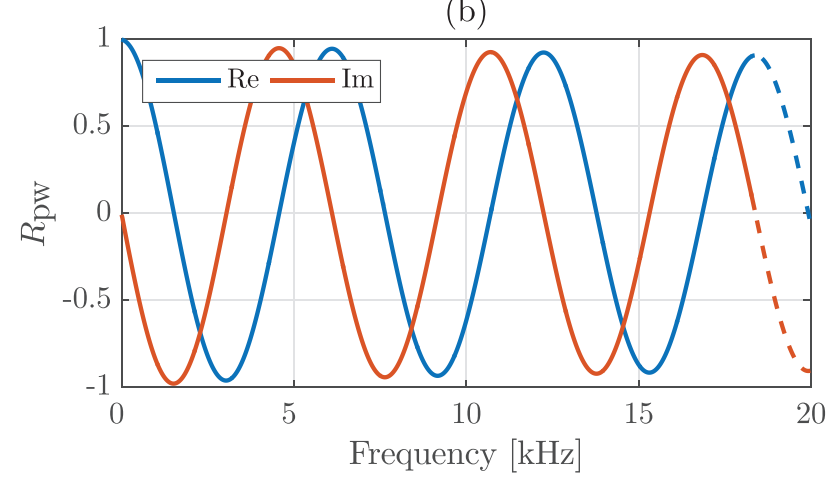

FIG. 2. (Color online) Analytically calculated real and imaginary parts of the (a) plane-wave acoustic impedance $Z_{\mathrm{pw}}$ [Eq. (32)] and (b) corresponding reflectance $R_{\text {pw }}$ [Eq. (33)] of a rigidly terminated waveguide of radius $a=2.35 \mathrm{~mm}$ and length $l=2.8 \mathrm{~cm}$. The dashed lines represent the discarded part of the spectrum above the truncation frequency $f_{\mathrm{t}}=18.3 \mathrm{kHz}$, in which the Hermitian-symmetric spectrum is replicated, to restore differentiability, and up to the maximum frequency $f_{\mathrm{m}}=20 \mathrm{kHz}$ of available data.

and sine, respectively, and thereby satisfy the constraints of strict causality.

Figures 2(a) and 2(b) show the real and imaginary parts of the analytically calculated plane-wave impedance $Z_{\mathrm{pw}}$ and the corresponding reflectance $R_{\mathrm{pw}}$ of a simulated waveguide of radius $a=2.35 \mathrm{~mm}$ and length $l=2.8 \mathrm{~cm}$. The data was calculated in frequency increments of $\Delta f=f_{\mathrm{s}} / N$ $=44.1 \mathrm{kHz} / 2048 \approx 21.5 \mathrm{~Hz}$ between $0 \mathrm{~Hz}$ and $20 \mathrm{kHz}$ for comparison with subsequent measurements in the evaluation waveguide. The figures also show the result of the truncation process, with the discarded part of the spectrum beyond the truncation frequency to restore differentiability, as the dashed lines. This yielded a truncation frequency of $f_{\mathrm{t}}$ $=18.3 \mathrm{kHz}$ as opposed to the available data up to the maximum frequency $f_{\mathrm{m}}=20 \mathrm{kHz}$.

Figures 3(a) and 3(b) show the real and imaginary impedance estimation errors $\operatorname{Re}\left\{\epsilon_{Z}\right\}$ and $\operatorname{Im}\left\{\epsilon_{Z}\right\}$, and timedomain reflectance $r$ of the plane-wave quantities of the simulated waveguide [Eq. (33)]. Time-domain reflectance is presented as it rather intuitively illustrates causality as opposed to $\epsilon_{Z}$. The figures confirm that this plane-wave impedance and reflectance are indeed causal, and that both $\operatorname{Re}\left\{\epsilon_{Z}\right\}$ and $\operatorname{Im}\left\{\epsilon_{Z}\right\}$ are close to zero. Figure 3(a) also illustrates the incapability of restoring perfect differentiability in the reflectance spectrum in terms of the slight deviant behavior in $\operatorname{Re}\left\{\epsilon_{Z}\right\}$ and $\operatorname{Im}\left\{\epsilon_{Z}\right\}$ towards the truncation frequency $f_{\mathrm{t}}=18.3 \mathrm{kHz}$. 

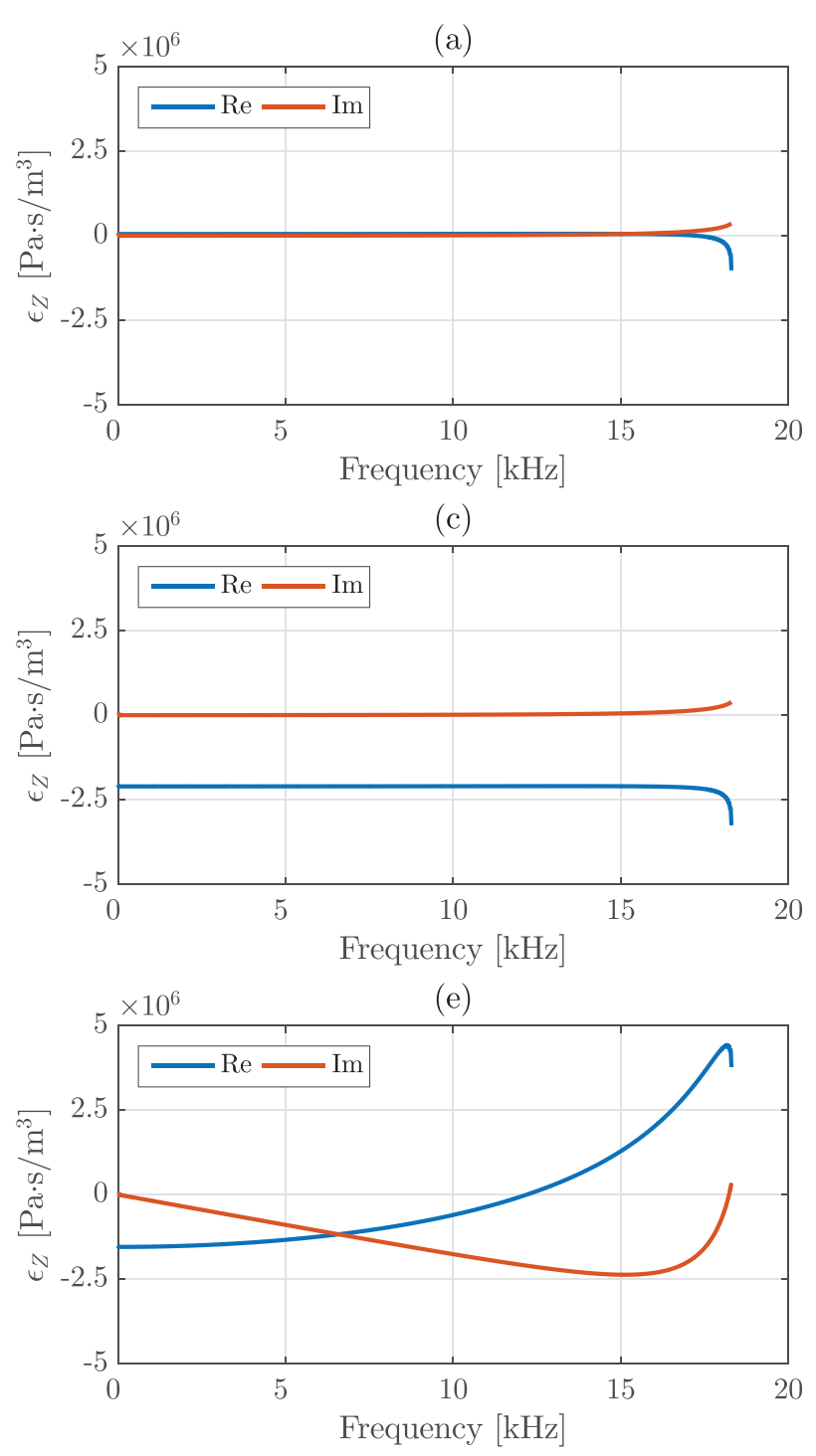

(b)

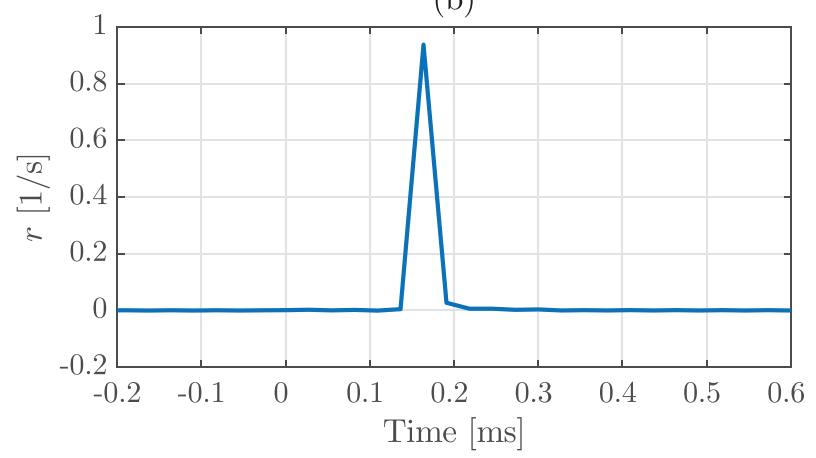

(d)

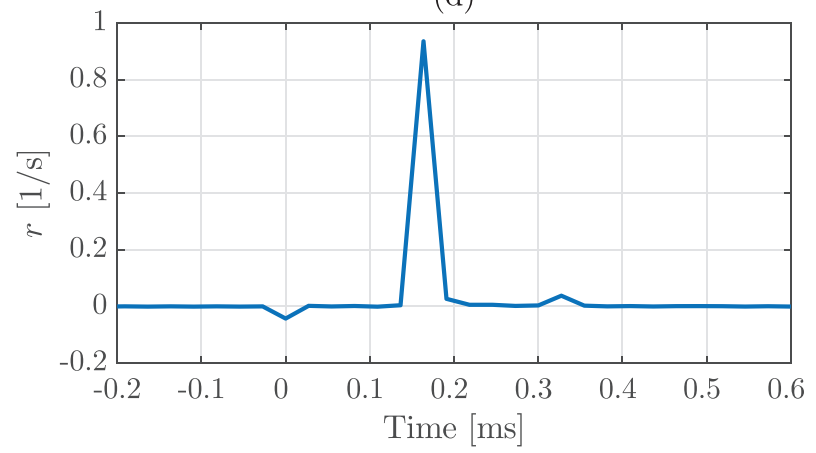

(f)

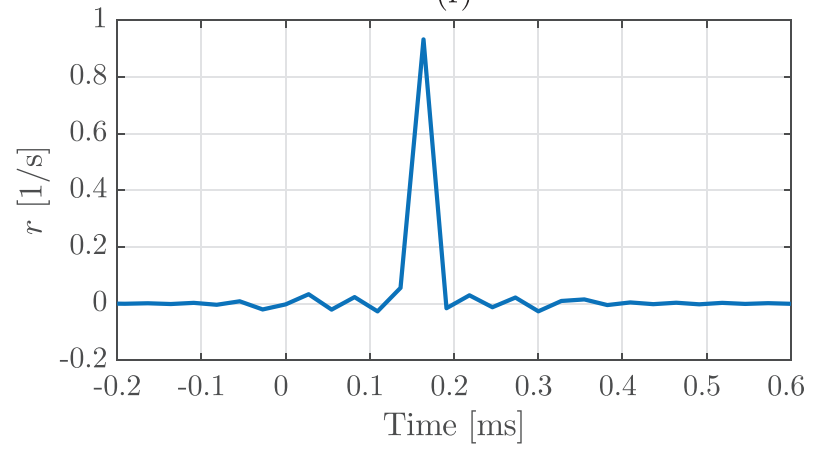

FIG. 3. (Color online) Analytically calculated (a), (c), (e) real and imaginary impedance estimation errors $\operatorname{Re}\left\{\epsilon_{Z}\right\}$ and $\operatorname{Im}\left\{\epsilon_{Z}\right\}$, and (b), (d), (f) time-domain reflectances $r$ of a rigidly terminated waveguide of characteristic impedance $Z_{0}=2.37 \times 10^{7} \mathrm{~Pa} \mathrm{~s} / \mathrm{m}^{3}(a=2.35 \mathrm{~mm})$ and length $l=2.8 \mathrm{~cm}$. (a), (b) The planewave quantities [Eq. (33)]. (c), (d) Utilizing an incorrect characteristic impedance $Z_{0}^{\prime}=2.59 \times 10^{7} \mathrm{~Pa} \mathrm{~s} / \mathrm{m}^{3}(a=2.25 \mathrm{~mm})$ for calculating reflectance [Eq. (16)]. (e), (f) Modeling evanescent modes using an acoustic inertance $L=-32.1 \mathrm{~kg} / \mathrm{m}^{4}$ [Eq. (11)], using the correct characteristic impedance $Z_{0}$.

Figures 3(c) and 3(d) show $\operatorname{Re}\left\{\epsilon_{Z}\right\}, \operatorname{Im}\left\{\epsilon_{Z}\right\}$, and $r$ using the plane-wave impedance, but introducing a mismatch in characteristic impedance in the calculation of reflectance $R_{\mathrm{pw}}^{\prime}$ as in Eq. (16), setting $Z_{0}^{\prime}=2.59 \times 10^{7} \mathrm{~Pa} \quad \mathrm{~s} / \mathrm{m}^{3}$ $(a=2.25 \mathrm{~mm})$. As expected, the incorrect characteristic impedance causes an offset in $\operatorname{Re}\left\{\epsilon_{Z}\right\}$, while $\operatorname{Im}\left\{\epsilon_{Z}\right\}$ is unaffected. For $t \leq 0$, it thus only contributes with an impulse in $t=0$ in the time-domain reflectance and agrees with the approach of Rasetshwane and Neely (2011) in estimating characteristic impedance in the absence of evanescent modes.

Finally, Figs. 3(e) and 3(f) show $\operatorname{Re}\left\{\epsilon_{Z}\right\}, \operatorname{Im}\left\{\epsilon_{Z}\right\}$, and $r$ using the correct characteristic impedance $Z_{0}$, but adding an acoustic inertance $j \omega L$ to the plane-wave input impedance [Eq. (11)] prior to calculating reflectance. The result thus mimics the effect of evanescent modes on a measured reflectance. The evanescent-modes inertance $L=-32.1 \mathrm{~kg} / \mathrm{m}^{4}$ was calculated analytically. It is clear that a degree of noncausality is introduced into impedance and reflectance. As expected, $\operatorname{Im}\left\{\epsilon_{Z}\right\}$ increases linearly with frequency except for a deviation at high frequencies, representing a half period of the smoothed sawtooth function similar to Fig. 1(b), though without the replicated spectrum. Additionally, an offset at $f=0$ and diverging behavior with increasing frequency due to the inertance are introduced into $\operatorname{Re}\left\{\epsilon_{Z}\right\}$, such that the characteristic impedance cannot be estimated in the presence of evanescent modes.

\section{EXPERIMENTAL RESULTS}

\section{A. Evaluation waveguide}

To illustrate the steps [see steps (1)-(3) of Sec. II E for details] of the compensation scheme of the proposed methodology and to compare with the simulation in Fig. 3, the acoustic probe was placed, without an ear tip, in the input plane of the evaluation waveguide $\left(Z_{0}=2.37 \times 10^{7} \mathrm{~Pa} \mathrm{~s} /\right.$ $\left.\mathrm{m}^{3}, a=2.35 \mathrm{~mm}\right)$. For this waveguide, the first higher-order mode starts to propagate at $f_{1,0}=42.7 \mathrm{kHz}$ with $\alpha_{1,0}^{\prime}=1.84$ 
[Eq. (12)]. Setting the initial value for the characteristic impedance to $Z_{0}^{\prime}=2.59 \times 10^{7} \mathrm{~Pa} \mathrm{~s} / \mathrm{m}^{3}(a=2.25 \mathrm{~mm})$, the compensation scheme has no preliminary knowledge of the features of the acoustic load under investigation.

Figures 4(a) and 4(b) show the real and imaginary impedance estimation errors $\operatorname{Re}\left\{\epsilon_{Z}\right\}$ and $\operatorname{Im}\left\{\epsilon_{Z}\right\}$, and timedomain reflectance $r$ resulting from step (1). Thus, the reflectance is calculated from $Z_{0}^{\prime}$ and without any compensation for evanescent modes. The figures indicate the presence of a significant non-causality in reflectance and incapability in correctly estimating the characteristic impedance.

Figures 4(c) and 4(d) show $\operatorname{Re}\left\{\epsilon_{Z}\right\}, \operatorname{Im}\left\{\epsilon_{Z}\right\}$, and $r$ resulting from step (2), estimating the evanescent-modes inertance $\hat{L}=-34.1 \mathrm{~kg} / \mathrm{m}^{4}$ [Eq. (29)] and subtracting it from the measured impedance [Eq. (15)]. This value is similar to the analytical value of $L=-32.1 \mathrm{~kg} / \mathrm{m}^{4}$ from Sec. III. The figures show a remaining error in $\operatorname{Re}\left\{\epsilon_{Z}\right\}$, similar to the analytical case in Figs. 3(c) and 3(d). This indicates that the reflectance measurement is now mainly affected by a mismatch in characteristic impedance.

Finally, Figs. 4(e) and 4(f) show $\operatorname{Re}\left\{\epsilon_{Z}\right\}, \operatorname{Im}\left\{\epsilon_{Z}\right\}$, and $r$ resulting from step (3), estimating the characteristic impedance $\hat{Z}_{0}=2.35 \times 10^{7} \mathrm{~Pa} \mathrm{~s} / \mathrm{m}^{3}(a=2.36 \mathrm{~mm})$ [Eq. (30)]. This value is in close agreement with $Z_{0}$ of the waveguide, with a relative error of 0.011 . The obtained $\operatorname{Re}\left\{\epsilon_{Z}\right\}$ and $\operatorname{Im}\left\{\epsilon_{Z}\right\}$ have attained levels comparable to the analytical case in Figs. 3(a) and 3(b), and the non-causality is also visually confirmed to have been significantly reduced from $r$. A small non-causal component is still present in the measurement, both seen in negative time in $r$ and at high frequencies in $\epsilon_{Z}$. This could be caused by small calibration errors towards higher frequencies or noise in the measurements. However, this does not appear to limit the capability of the methodology in this case.

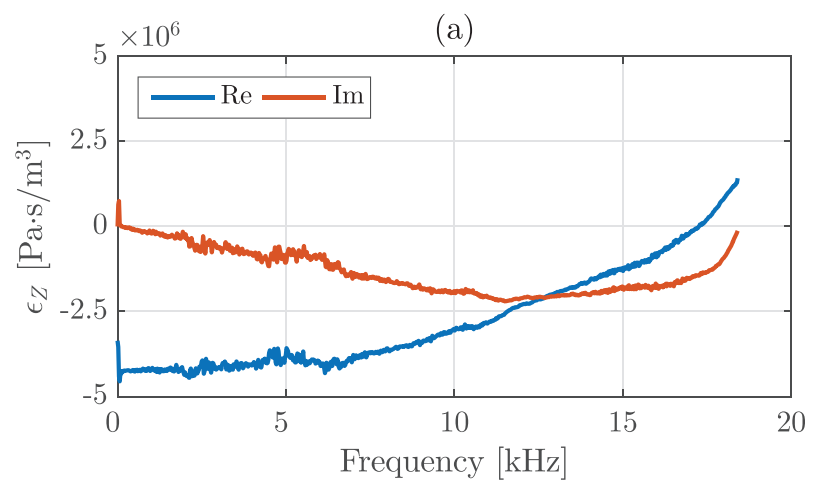

(c)

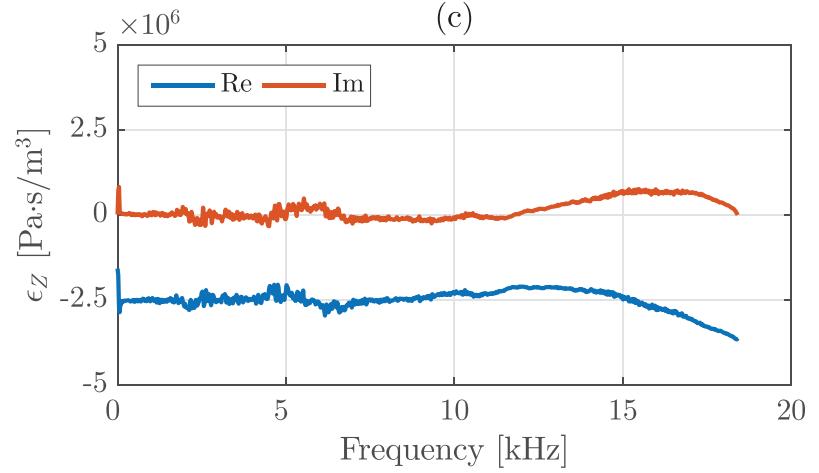

(e)

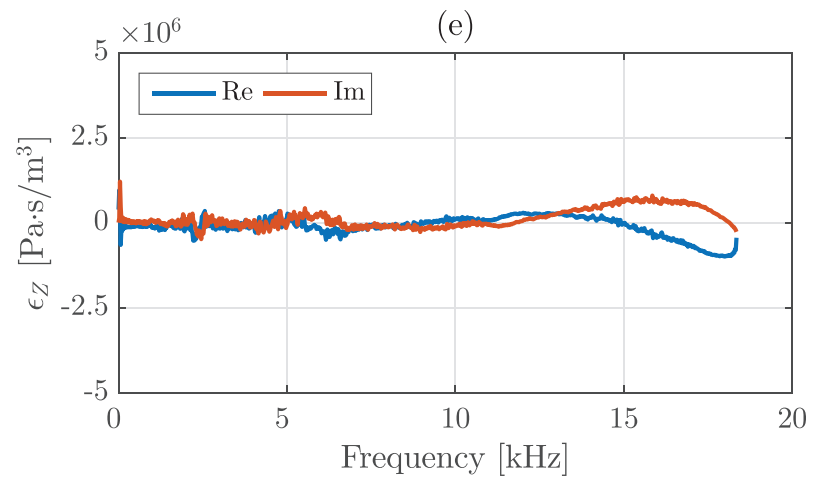

(b)

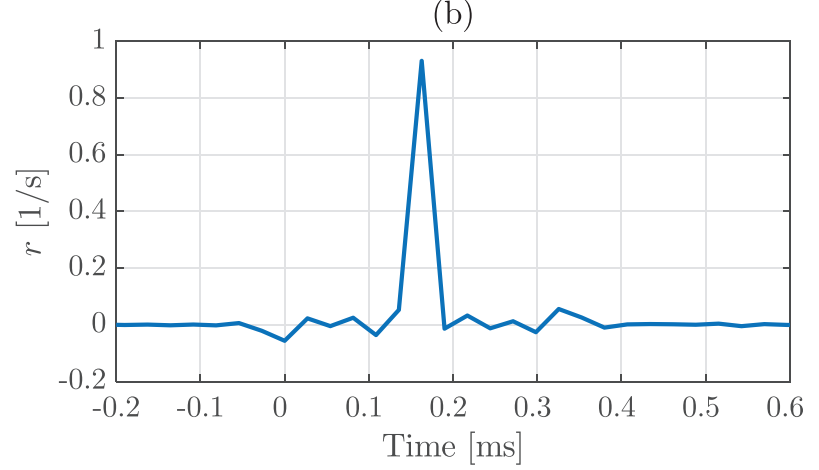

(d)

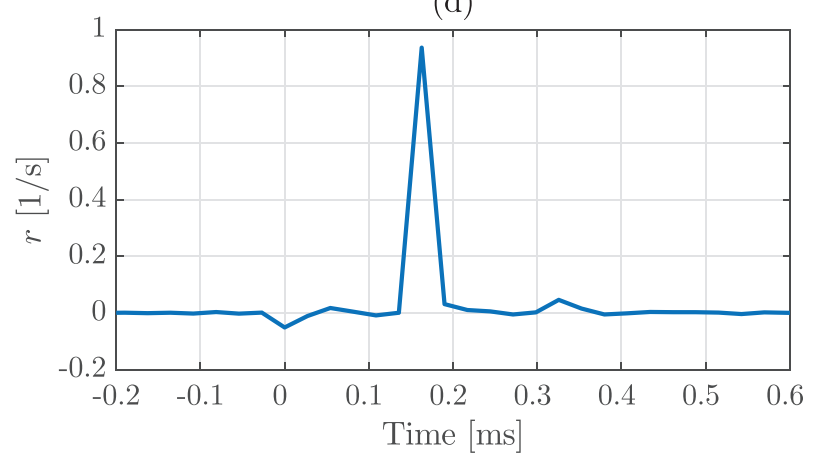

(f)

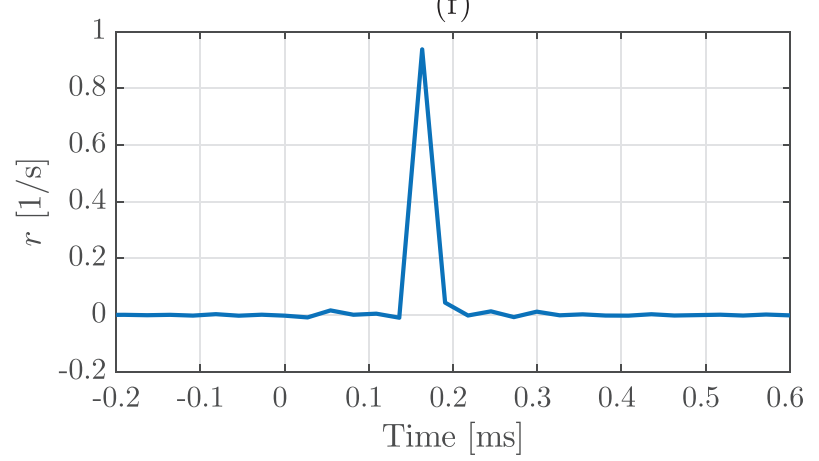

FIG. 4. (Color online) Measured (a), (c), (e) real and imaginary impedance estimation errors $\operatorname{Re}\left\{\epsilon_{Z}\right\}$ and $\operatorname{Im}\left\{\epsilon_{Z}\right\}$, and (b), (d), (f) time-domain reflectances $r$ of a rigidly terminated waveguide of characteristic impedance $Z_{0}=2.37 \times 10^{7} \mathrm{~Pa} \mathrm{~s} / \mathrm{m}^{3}(a=2.35 \mathrm{~mm})$, length $l=2.8 \mathrm{~cm}$, and analytically calculated evanescent-modes inertance $L=-32.1 \mathrm{~kg} / \mathrm{m}^{4}$ resulting from steps (1)-(3); see Sec. IIE for details. (a), (b) The result of step (1), the measurement without any compensation for evanescent modes and an initial value $Z_{0}^{\prime}=2.59 \times 10^{7} \mathrm{~Pa} \mathrm{~s} / \mathrm{m}^{3}(a=2.25 \mathrm{~mm})$ for calculating reflectance. (c), (d) The result of step (2), having estimated the acoustic inertance $\hat{L}=-34.1 \mathrm{~kg} / \mathrm{m}^{4}$ [Eq. (29)] to compensate for evanescent modes, still using $Z_{0}^{\prime}$. (e), (f) The result of step (3), additionally having estimated $\hat{Z}_{0}=2.35 \times 10^{7} \mathrm{~Pa} \mathrm{~s} / \mathrm{m}^{3}(a=2.36 \mathrm{~mm})$ [Eq. (30)]. 


\section{B. Ear simulator}

To further demonstrate the capability of the proposed methodology and its effect on a measurement of impedance and reflectance in a load similar to the human ear canal, the probe was positioned in the ear simulator $\left(Z_{0}=9.32 \times 10^{6}\right.$ $\mathrm{Pa} \mathrm{s} / \mathrm{m}^{3}, a=3.75 \mathrm{~mm}$ ) using the ear tip. For the ear simulator, the first higher-order mode starts to propagate at $f_{1,0}$ $=26.8 \mathrm{kHz}$ with $\alpha_{1,0}^{\prime}=1.84$ [Eq. (12)]. The results of the steps [see steps (1)-(3) of Sec. IIE for details] in the proposed compensation scheme were obtained using an initial value for the characteristic impedance $Z_{0}^{\prime}=7.25 \times 10^{6} \mathrm{~Pa} \mathrm{~s} /$ $\mathrm{m}^{3}(a=4.25 \mathrm{~mm})$. This value was chosen to be representative of a mismatch one might encounter in a real ear, assuming an invariant characteristic impedance $Z_{0}$. Step (2) estimated an acoustic inertance $\hat{L}=31.5 \mathrm{~kg} / \mathrm{m}^{4}$ [Eq. (29)], which is in good agreement with the analytically calculated value $L=29.6 \mathrm{~kg} / \mathrm{m}^{4}$. Step (3) estimated a characteristic impedance $\hat{Z}_{0}=9.25 \times 10^{6} \mathrm{~Pa} \mathrm{~s} / \mathrm{m}^{3} \quad(a=3.76 \mathrm{~mm})$ [Eq. (30)], corresponding to a relative error of 0.0066 .

Figures 5(a) and 5(b) show the impedance magnitudes $|Z|$ and phases $\angle Z$ obtained from steps (1)-(3). As the estimation of characteristic impedance does not affect the impedance measure itself, the results of steps (2) and (3) are identical. The figures mainly show the translation in the position of impedance minima due to the evanescent-modes inertance $\hat{L}$.

Figures 5(c) and 5(d) show the reflectance magnitude $|R|$ and the reflectance group delay $\tau_{\mathrm{g}}=-d \angle R / d \omega$. $\tau_{\mathrm{g}}$ was convolved by a 19-point Blackman window to reduce noise. The figures show that evanescent modes affect $|R|$ and $\tau_{\mathrm{g}}$ in step (1) increasingly with frequency compared to step (2). Conversely, estimating $\hat{Z}_{0}$ in step (3) introduces variation across the entire frequency range compared to step (2), but most distinct in points where $|R|$ is small, resulting in a much smoother curve across the frequency range. Also, at low frequencies where the load is compliance dominated, $\hat{Z}_{0}$ has a large effect on $\tau_{\mathrm{g}}$.

Finally, Figs. 5(e) $-5(\mathrm{~g})$ show the real and imaginary impedance estimation errors $\operatorname{Re}\left\{\epsilon_{Z}\right\}$ and $\operatorname{Im}\left\{\epsilon_{Z}\right\}$, and timedomain reflectances $r$, respectively. The behavior resulting from the steps (1)-(3) is similar to the simulation in Fig. 3 and the results in Fig. 4, and the final result indicates that the non-causality has been significantly reduced in the impedance and reflectance. It is interesting to also note that, while $\hat{Z}_{0}$ mainly affects $r$ in $t=0$, evanescent modes have a large influence on $r$ before and after the main reflection of the ear simulator. These measurements also show a degree of assumed calibration errors or system noise towards higher frequencies, however without affecting the methodology.

\section{DISCUSSION}

In hearing diagnostics and other fields, evanescent modes and incorrect estimates of characteristic impedance introduce errors and uncertainties into measurements of reflectance and impedance. The compensation for evanescent modes using an acoustic inertance and estimation of characteristic impedance to reduce the non-causality in the reflectance using the Hilbert transform appears to address most of these errors. Despite these errors, existing reflectance and impedance-based methods for calibrating stimulus levels in situ appear to perform well. However, given the results in Fig. 5, the errors in stimuli to the ear would probably only amount to a few $\mathrm{dBs}$, which is also in line with the results of Brass and Locke (1997). It is thus likely that the proposed methodology would only result in minor performance improvements in methods for calibrating stimulus levels. Conversely, the capability of ear canal reflectance in identifying specific conductive pathologies could be limited by evanescent modes and, in particular, incorrect characteristic impedance. While the present study does not demonstrate that the proposed methodology can provide accurate measurements of impedance and reflectance in real ears, it does give an indication of the implications of incorrect characteristic impedance and evanescent modes, and how these effects can degrade the reflectance measure. The results of this study indicate that the reflectance above approximately $3 \mathrm{kHz}$ does not provide valid information on the conductive capabilities of the middle ear, unless at least the characteristic impedance can be accurately estimated, which is similar to the conclusion of Schmidt and Hudde (2009). The proposed methodology could thus provide a step forward in improving the sensitivity and specificity in identifying and distinguishing such pathologies, and provide minor improvements in calibrating stimulus levels. However, more research is required to systematically evaluate the capability of the method in real ears in a clinical setup.

The proposed methodology appears to perform well in the utilized evaluation waveguide and ear simulator in estimating the characteristic impedance and evanescent-modes inertance. Scheperle et al. (2011) reported the estimated characteristic impedances in evaluation waveguides of various radii using the method of Rasetshwane and Neely (2011) and Rasetshwane et al. (2012) with errors within a few percent. However, it is interesting to note that the accuracy of their estimated characteristic impedances decreases with an increasing mismatch between calibration and evaluation waveguide radii [see Table II of Scheperle et al. (2011)]. The proposed method was evaluated in waveguides with radii very different from the calibration waveguides but still appears to perform better. A comparison with the method and errors reported by Keefe et al. (1992) is not valid due to the limitations associated with the finite frequency range discussed in Sec. IA. The calibration method described by Nørgaard et al. (2017) and utilized in this paper does not include the material properties of the ear tip in the source parameters. The ear tip therefore appears as a parallel component to a measured impedance, and could likely contribute with a non-causal component to the measurement. Besides small calibration errors and noise, the ear tip could be a reason for the variations observed in Figs. 5(e) and 5(f) towards higher frequencies, though the effects of evanescent modes and characteristic impedance appear to be much more profound. The results suggest that the material properties of the specific utilized ear tip do not influence the proposed methodology in any significant way.

The proposed methodology relies on two important assumptions, namely that the characteristic impedance is real 
(a)

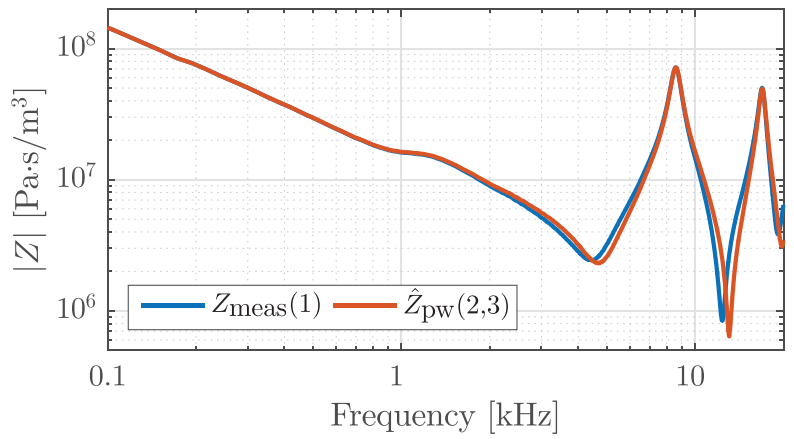

(c)

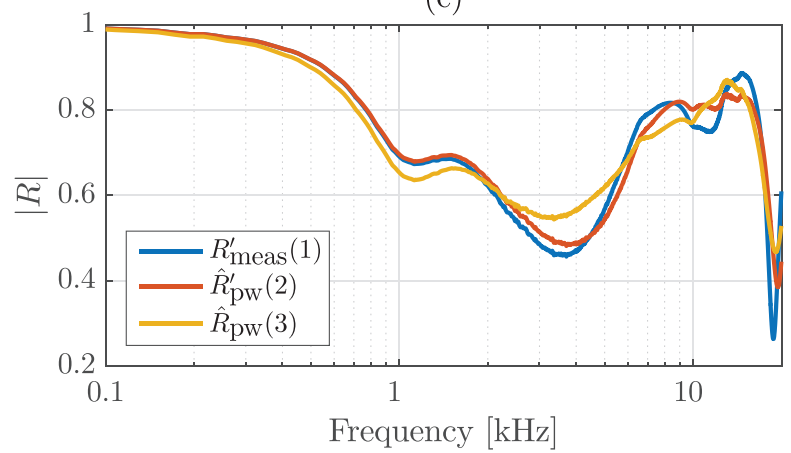

(e)

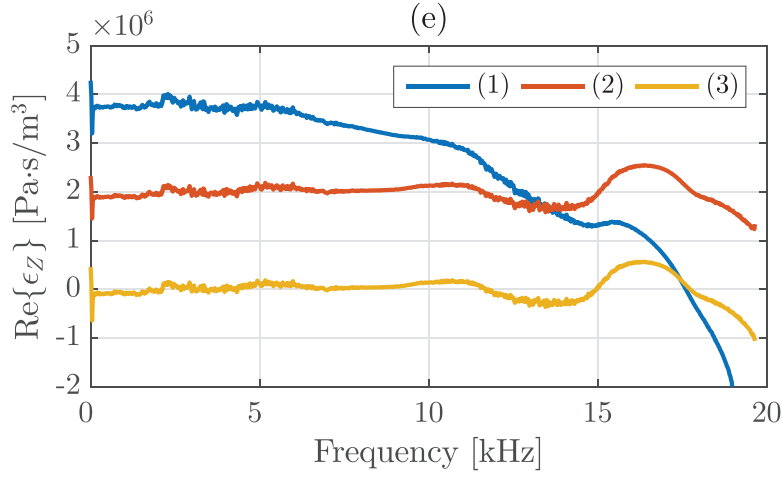

(b)

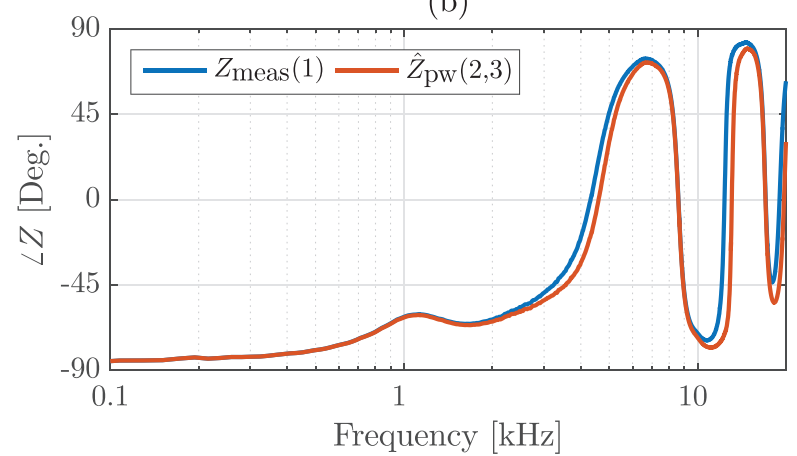

(d)

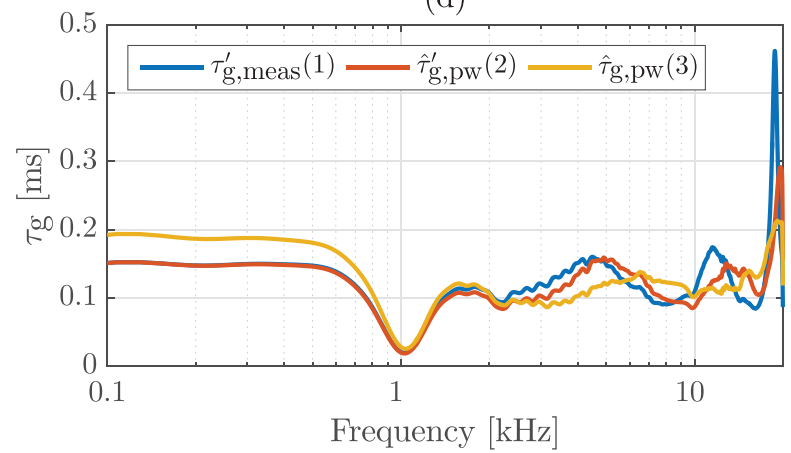

(f)

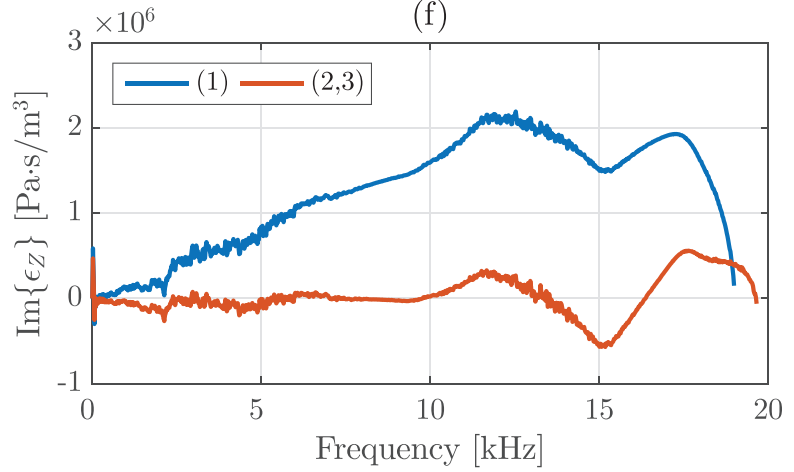

(g)

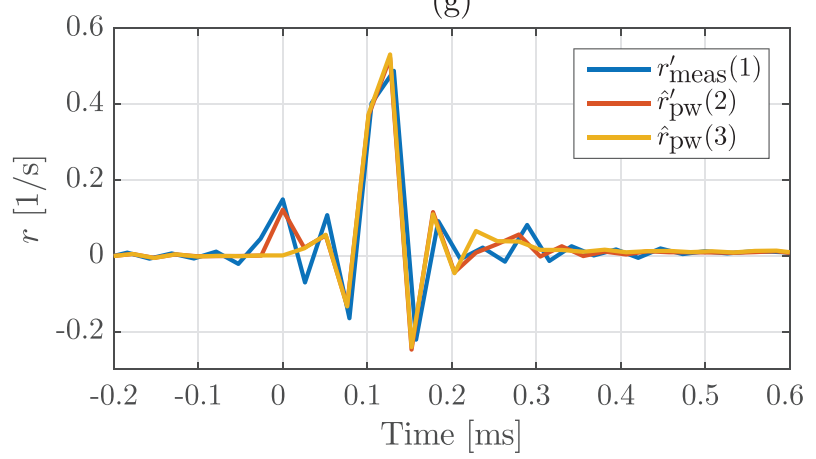

FIG. 5. (Color online) The results of the steps [see steps (1)-(3) of Sec. IIE for details] of the proposed compensation scheme on the measurement in the occluded-ear simulator $\left(Z_{0}=9.32 \times 10^{6} \mathrm{~Pa} \mathrm{~s} / \mathrm{m}^{3}, a=3.75 \mathrm{~mm}\right.$, and $\left.L=29.6 \mathrm{~kg} / \mathrm{m}^{4}\right)$, setting $Z_{0}^{\prime}=7.25 \times 10^{6} \mathrm{~Pa} \mathrm{~s} / \mathrm{m}^{3}(a=4.25 \mathrm{~mm})$. Step $(2)$ estimated the evanescent-modes inertance $\hat{L}=31.5 \mathrm{~kg} / \mathrm{m}^{4}$, and step (3) the characteristic impedance $\hat{Z}_{0}=9.25 \times 10^{6} \mathrm{~Pa} \mathrm{~s} / \mathrm{m}^{3}(a=3.76 \mathrm{~mm})$. (a), (b) The impedance magnitudes $|Z|$ and phases $\angle Z$. (c), (d) The reflectance magnitudes $|R|$ and group delays $\tau_{\mathrm{g}}$. (e), (f) Real and imaginary impedance estimation errors Re $\left\{\epsilon_{Z}\right\}$ and $\operatorname{Im}\left\{\epsilon_{Z}\right\}$, and (g) time-domain reflectances $r .|Z|, \angle Z$, and $\operatorname{Im}\left\{\epsilon_{Z}\right\}$ are identical for steps (2) and (3).

and frequency independent, and that the true plane-wave reflectance is strictly causal, i.e., no impulses in $t=0$ $(\operatorname{Re}\{R(\infty)\}=0)$. These assumptions fall short in the case of a non-uniformity of the waveguide at the position of the probe, which is the case for an oblique probe insertion into a waveguide or an ear canal. Such oblique probe insertion shoves the probe tube closer to one ear-canal wall at an angle, which acoustically appears as a rapidly expanding horn just in front of the probe. The consequence of this horn gradient when applying the proposed methodology is that the superposition of the evanescent-modes and horn inertances (Rasetshwane and Neely, 2011; Rasetshwane et al., 2012) is 
obtained. For the characteristic impedance, assuming it is frequency independent and real, a value is obtained corresponding to the apparent cross-sectional area just in front of the probe. Thus, the obtained characteristic impedance varies with the obliqueness of the probe insertion, and this can reduce the accuracy and test-retest variability of the reflectance. In addition, variations in the attachment of the ear tip to the probe can cause similar issues in the measured impedance and reflectance. When applying the proposed methodology in ear-canal measurements, one should exhibit caution in positioning the probe perpendicular to the input plane of the probe tip in the ear canal. Also, the ear tip should be attached to the probe in a way such that the tip of the probe tube and the ear tip, as far as possible, are aligned in a single plane. However, more research is required to overcome these challenges, perhaps in the form of additional compensation or ear tips that facilitate a more precise probe placement.

The proposed methodology also relies on the assumption that the imaginary addition from evanescent modes to the input impedance is proportional to frequency. This assumption is invalid when the frequency approaches the cut-on frequency of the first higher-order propagating mode in the waveguide [Eq. (12)]. Given the radii of the evaluation waveguide and ear simulator utilized in this study, the first higher-order modes start to propagate well above the investigated frequency range $\left(f_{1,0}=42.7 \mathrm{kHz}\right.$ and $f_{1,0}=26.8 \mathrm{kHz}$, respectively, with $f_{\mathrm{m}}=20 \mathrm{kHz}$ ). It is difficult to estimate at which frequency the approximation using an inertance to quantify evanescent modes is insufficient, and it probably depends on the application, e.g., reflectance measurements or stimulus-level calibration. In any case, the approximation as an inertance is likely to be superior to no compensation, and otherwise the maximum frequency $f_{\mathrm{m}}$ can simply be reduced to avoid such variation. In order to restore differentiability in the reflectance spectrum, its real and imaginary parts must exhibit a harmonic behavior. This is always the case when the majority of sound is reflected by a waveguide termination and thus mainly delayed by the round-trip time $t=2 l / c$. If the waveguide is short enough or the maximum frequency low enough that $l<c /\left(4 f_{\mathrm{m}}\right)$, differentiability cannot be restored as the reflectance does not contain one half oscillation between $f=0$ and $f=f_{\mathrm{m}}$, and this severely limits the application of the proposed method. Nonuniformities in the ear canal could degrade this harmonic behavior, but presumably not enough to render the method inapplicable as the tympanic membrane constitutes a major impedance discontinuity. While the truncation process avoids most errors due to the finite frequency range, potential errors beyond the truncation frequency cannot be observed in the impedance estimation errors and timedomain reflectance. In addition, when a large mismatch between the characteristic-impedance initial value $Z_{0}^{\prime}$ and the true characteristic impedance of the waveguide $Z_{0}$ is present, a non-smooth behavior, similar to that of the impedance, forms in the real and imaginary parts of reflectance. If this mismatch becomes too large, instabilities can occur in the discrete Hilbert transform. The limit where this occurs is defined by many factors such as the fast Fourier transformsize, waveguide length, and damping in the load. In loads similar to the human ear canal, this does not appear to pose a problem, even for mismatches much larger than investigated in this study.

A current unresolved uncertainty, potentially affecting the proposed methodology, is its dependency on system noise. Although the presented measurements were not carried out in a sound booth, they seem to be affected by noise by only a small degree, compared to the potential noise induced by, e.g., head movements in real ears. Calculating the time-domain reflectance using the inverse Fourier transform constitutes an ill-posed inverse problem. If the measurements are affected by noise, it can contribute to the derived time-domain quantities in any points in time, potentially introducing a non-causality. Currently, no further assessment of the potential influence of noise on the proposed methodology has been conducted, but such investigation should be carried out before the methodology is employed in potentially noisy applications. In addition to noise, the ear cannot be assumed to be a passive system. Burns et al. (1998) showed that energy reflectance can exceed unity near spontaneous otoacoustic-emission frequencies. However, no further investigation has been conducted to assess the potential effect of active mechanisms in the ear on the proposed methodology.

In some ideal cases, it may be possible to estimate the characteristic impedance and evanescent-modes inertance without utilizing the Hilbert transform. This could be achieved by selecting $Z_{0}$ and $L$ such that the time-domain reflectance $r$ is minimized for $t \leq 0$. However, a measured time-domain reflectance, derived from an inverse Fourier transform of frequency-domain data, is rarely as wellbehaved as the considered measurements in Figs. 4 and 5. If frequency-domain windowing is applied to the reflectance prior to calculating the inverse Fourier transform (Rasetshwane and Neely, 2011), the confined effect of incorrect characteristic impedance in $t=0$ is smeared in time. This renders the effect indistinguishable from the effect of evanescent modes, which may affect points for $t \leq 0$, and possibly also actual reflections for $t>0$ from the ear canal. As briefly discussed in Sec. II D, a lack of differentiability in the reflectance essentially convolves the time-domain reflectance with a sinc function. This is the effect that Rasetshwane et al. (2012) refers to as "Gibbs phenomena." For the measured time-domain reflectances in Figs. 4 and 5, caution was taken to place the probe accurately, and the measurements were obtained immediately after the calibration procedure such that there was no drift of the Thévenin source parameters. Such drifts in the source parameters and other measurement errors can cause an incapability in restoring differentiability in the reflectance spectrum through truncation. These effects appear close to the actual signal in the time-domain reflectance and can obscure the effects of incorrect characteristic impedance and evanescent modes. However, in the impedance estimation errors, calculated from the Hilbert transform, the effects will be confined towards higher frequencies, and can thus be avoided by restricting the frequency range over which quantities are analyzed. Furthermore, a different truncation frequency could be utilized for the real and imaginary parts to reduce 
such effects. Still, more research would be beneficial to systematically assess the effect of instability in the Thévenin source parameters on the proposed methodology and existing time-domain measures.

\section{CONCLUSION}

A method has been proposed that can accurately estimate the acoustic inertance accounting for evanescent modes and the characteristic impedance, based on a measurement of the input impedance of a waveguide using an acoustic probe. The method determines these quantities such that the non-causality in the reflectance is minimized in the frequency domain using the Hilbert transform and thereby compensates for the effects of evanescent modes and incorrect characteristic impedance. The method is applicable in waveguides that are uniform at the position of the acoustic probe and could provide a step forward in increasing the validity of ear-canal impedance and reflectance measurements, and stimulus level calibration. It has been demonstrated how an evanescent-modes inertance and a characteristic-impedance mismatch theoretically affect the causality of reflectance and how these parameters can be determined for a given degree of non-causality. The behavior was confirmed, both from an analytical case and actual measurements in an evaluation waveguide and an occluded-ear simulator, using an ear probe. The evanescent-modes inertances and the characteristic impedances were estimated, both in close agreement with the analytical values of the waveguides.

\section{ACKNOWLEDGMENTS}

The authors would like to thank the two anonymous reviewers for comments and suggestions for the manuscript. This research was funded by Interacoustics $\mathrm{A} / \mathrm{S}$ and the Innovation Fund Denmark under File No. 5189-00100B.

\section{${ }^{1}$ Information available at www.playrec.co.uk (Last viewed 12 November 2017).}

Blackstock, D. T. (2000). "Cylindrical waves," in Fundamentals of Physical Acoustics (Wiley, New York), Chap. 11, pp. 386-413.

Brass, D., and Locke, A. (1997). "The effect of the evanescent wave upon acoustic measurements in the human ear canal," J. Acoust. Soc. Am. 101, 2164-2175.

Burns, E. M., Keefe, D. H., and Ling, R. (1998). "Energy refectance in the ear canal can exceed unity near spontaneous otoacoustic emission frequencies," J. Acoust. Soc. Am. 103, 462-474.

Dalmont, J.-P., Curtit, M., and Yahaya, A. F. (2012). "On the accuracy of bore reconstruction from input impedance measurements: Application to bassoon crook measurements," J. Acoust. Soc. Am. 131, 708-714.

Egolf, D. P., Nelson, D. K., Howell, H. C., and Larson, V. D. (1993). "Quantifying ear-canal geometry with multiple computer-assisted tomographic scans," J. Acoust. Soc. Am. 93, 2809-2819.

Ellison, J. C., Gorga, M., Cohn, E., Fitzpatrick, D., Sanford, C. A., and Keefe, D. H. (2012). "Wideband acoustic transfer functions predict middle-ear effusion," Laryngoscope 122, 887-894.

Feeney, M. P., and Keefe, D. H. (2001). "Estimating the acoustic reflex threshold from wideband measures of reflectance, admittance, and power," Ear Hear. 22, 316-332.

Fletcher, N. H., and Rossing, T. D. (1991). The Physics of Musical Instruments (Springer-Verlag, New York).

Fletcher, N. H., Smith, J., Tarnopolsky, A. Z., and Wolfe, J. (2005). "Acoustic impedance measurements-Correction for probe geometry mismatch,” J. Acoust. Soc. Am. 117, 2889-2895.
Hsu, H. P. (1967). "Causality and the Hilbert transform," in Fourier Analysis, revised ed. (Simon \& Schuster, New York), pp. 239-243.

Huang, G. T., Rosowski, J. J., Puria, S., and Peake, W. T. (2000). "A noninvasive method for estimating acoustic admittance at the tympanic membrane," J. Acoust. Soc. Am. 108, 1128-1146.

Karal, F. C. (1953). "The analogous acoustical impedance for discontinuities and constrictions of circular cross section," J. Acoust. Soc. Am. 25, 327-334.

Keefe, D. H. (1984). "Acoustical wave propagation in cylindrical ducts: Transmission line parameter approximations for isothermal and nonisothermal boundary conditions," J. Acoust. Soc. Am. 75, 58-62.

Keefe, D. H., and Abdala, C. (2007). "Theory of forward and reverse middle-ear transmission applied to otoacoustic emissions in infant and adult ears," J. Acoust. Soc. Am. 121, 978-993.

Keefe, D. H., and Benade, A. H. (1981). "Impedance measurement source and microphone proximity effects," J. Acoust. Soc. Am. 69, 1489-1495.

Keefe, D. H., Folsom, R. C., Gorga, M. P., Vohr, B. R., Bulen, J. C., and Norton, S. J. (2000). "Identification of neonatal hearing impairment: Earcanal measurements of acoustic admittance and reflectance in neonates," Ear Hear. 21, 443-461.

Keefe, D. H., Ling, R., and Bulen, J. C. (1992). "Method to measure acoustic impedance and reflection coefficient," J. Acoust. Soc. Am. 91, 470-485.

Keefe, D. H., Sanford, C. A., Ellison, J. C., Fitzpatrick, D. F., and Gorga, M. P. (2012). "Wideband aural acoustic absorbance predicts conductive hearing loss in children,” Int. J. Audiol. 51, 880-891.

Lewis, J. D., McCreery, R. W., Neely, S. T., and Stelmachowicz, P. G. (2009). "Comparison of in-situ calibration methods for quantifying input to the middle ear," J. Acoust. Soc. Am. 126, 3114-3124.

McCreery, R. W., Pittman, A., Lewis, J., Neely, S. T., and Stelmachowicz, P. G. (2009). "Use of forward pressure level to minimize the influence of acoustic standing waves during probe-microphone hearing-aid verification," J. Acoust. Soc. Am. 126, 15-24.

Merchant, G. R., Röösli, C., Niesten, M. E. F., Hamade, M. A., Lee, D. J., McKinnon, M. L., Ulku, C. H., Rosowski, J. J., Merchant, S. N., and Nakajima, H. H. (2014). "Power reflectance as a screening tool for the diagnosis of superior semicircular canal dehiscence," Otol. Neurotol. 36, 172-177.

Munjal, M. L. (2014). Acoustics of Ducts and Mufflers, 2nd ed. (Wiley, Chichester, UK).

Nørgaard, K. R., Fernandez-Grande, E., and Laugesen, S. (2017). "Incorporating evanescent modes and flow losses into reference impedances in acoustic Thévenin calibration," J. Acoust. Soc. Am. 142, 3013-3024.

Papoulis, A. (1962). "Hilbert transforms," in The Fourier Integral and its Applications (McGraw-Hill, New York), pp. 198-201.

Piskorski, P., Keefe, D. H., Simmons, J. L., and Gorga, M. P. (1999). "Prediction of conductive hearing loss based on acoustic ear-canal response using a multivariate clinical decision theory," J. Acoust. Soc. Am. 105, 1749-1764.

Rasetshwane, D. M., and Neely, S. T. (2011). "Inverse solution of ear-canal area function from reflectance," J. Acoust. Soc. Am. 130, 3873-3881.

Rasetshwane, D. M., Neely, S. T., Allen, J. B., and Shera, C. A. (2012). "Reflectance of acoustic horns and solution of the inverse problem," J. Acoust. Soc. Am. 131, 1863-1873.

Scheperle, R. A., Goodman, S. S., and Neely, S. T. (2011). "Further assessment of forward pressure level for in situ calibration," J. Acoust. Soc. Am. 130, 3882-3892.

Scheperle, R. A., Neely, S. T., Kopun, J. G., and Gorga, M. P. (2008). "Influence of in situ, sound-level calibration on distortion-product otoacoustic emission variability,” J. Acoust. Soc. Am. 124, 288-300.

Schmidt, S., and Hudde, H. (2009). "Accuracy of acoustic ear canal impedances: Finite element simulation of measurement methods using a coupling tube," J. Acoust. Soc. Am. 125, 3819-3827.

Siegel, J. H., and Neely, S. T. (2017). "Eartip modification greatly reduces evanescent waves," in Proceedings of the 40th MidWinter Meeting, February 11-15, Baltimore, MD, pp. 55-56.

Souza, N. N., Dhar, S., Neely, S. T., and Siegel, J. H. (2014). "Comparison of nine methods to estimate ear-canal stimulus levels," J. Acoust. Soc. Am. 136, 1768-1787.

Voss, S. E., and Allen, J. B. (1994). "Measurement of acoustic impedance and reflectance in the human ear canal," J. Acoust. Soc. Am. 95, 372-384.

Withnell, R. H., Jeng, P. S., Waldvogel, K., Morgenstein, K., and Allen, J. B. (2009). "An in situ calibration for hearing thresholds," J. Acoust. Soc. Am. 125, 1605-1611. 